\title{
Las emociones en la vida universitaria: análisis de la relación entre autoconocimiento emocional y autorregulación emocional en adolescentes y jóvenes universitarios
}

\section{Emotions in university life: Analysis of the relationship between emotional self-awareness and emotional self- regulation in teens and college-aged students}
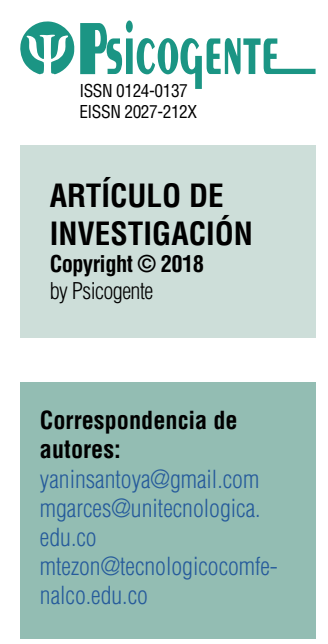

Recibido: $19-05-17$ Aceptado: 20-10-17 Publicado: $01-07-18$

Cómo citar este artículo (APA):

Santoya Montes, Y., Garcés Prettel, M. \& Tezón Boutureira, M. (2018). Las emociones en la vida universitaria: análisis de la relación entre autoconocimiento emocional y autorregulación emocional en adolescentes y jóvenes universitarios. Psicogente 21(40), 422-439. https://doi.org/10.17081/psico.21.40.3081

\section{Resumen}

Objetivo: Se presentan los resultados de una investigación correlacional que estableció la relación entre autoconocimiento y autorregulación emocional en universitarios colombianos.

Método: La muestra estuvo compuesta por 356 sujetos entre 15 y 22 años, que cursaban carreras profesionales en tres universidades de Cartagena de Indias (Colombia). La información fue recolectada a través de la escala CARE, con una consistencia interna de 0.722 para autoconocimiento y 0.750 para autorregulación.

Resultados: Los hallazgos revelan una asociación estadísticamente significativa entre los niveles de autoconocimiento emocional y los de autorregulación emocional de los participantes, encontrándose que a mayor percepción e identificación de las emociones, mayor es la probabilidad de desarrollar tolerancia a la frustración. De igual manera, se halló que la capacidad de hablar abiertamente de las emociones y de reconocer señales emocionales internas aumenta la probabilidad de regular los impulsos emocionales, de generar estrategias de afrontamiento y de desarrollar tolerancia a la frustración.

Conclusión: La comunicación y el reconocimiento de las emociones son aspectos determinantes para que estos jóvenes puedan regular sus emociones y enfrentar o manejar adecuadamente los problemas cotidianos.

Palabras clave: inteligencia emocional, conciencia emocional, autoconocimiento emocional,regulación emocional, comunicación emocional.

Abstract:

Objective: This paper aims to submit a correlational research in order to establish a relationship between emotional self-awareness and emotional self-regulation in Colombian university students.

Method: 356 university students aged between 15 and 22 from Cartagena (Colombia) were sampled. CARE scale was used to collect the information, giving an internal consistency of 0.722 to 0.750 for self-knowledge and self-regulation.

Results: The findings reveal a statistically significant association between participants' levels of emotional self-awareness and emotional self-regulation. A higher perception and identification of emotions was found, also the greater the likelihood of developing tolerance to the frustration. Similarly, the capability to talk openly about emotions and inner emotional signals increases the likelihood of regulating emotional impulsiveness, to generate coping strategies and to have greater tolerance to frustration, was established

Conclusions: Emotions communication and recognition are determining factors so that these young people can regulate their emotions and face or handle better the daily problems.

Keywords: emotional intelligence, emotional awareness, emotional self-knowledge, emotional regulation, emotional communication. 


\section{INTRODUCCIÓN}

En los últimos veinte años se ha desarrollado un interés creciente en la comunidad científica en torno a la influencia de las habilidades emocionales y, de manera concreta, en la manera cómo se relacionan los individuos y cómo afrontan los conflictos y las presiones diarias (Ariza, Bonilla, León y Vaca, 2017; Extremera, Férnandez \& Salovey, 2006; Rieffe, Broekhof, Kouwenberg, Faber, Tsutsui \& Güroğlu, 2016).

El Autoconocimiento Emocional ( $A E$ ) y la Autorregulación Emocional (ARE) son precisamente indicadores de dicha inteligencia emocional, y hacen referencia a todas las habilidades que permiten el control y la expresión emocional (Estrada, 2015; Goleman, 2012; Valles \& Valles, 2003; Salovey \& Mayer, 1990). Estos aspectos de la inteligencia emocional son sustanciales para consolidar la autoconfianza, la cual es un factor relacionado positivamente con el bienestar y la felicidad (Szczygieł \& Mikolajczak, 2017), que a su vez contribuyen a que los individuos lleven una vida personal y social más placentera. Asimismo, el AE y la ARE ayudan a: reducir la ansiedad, superar situaciones estresantes, mejorar las relaciones interpersonales, tolerar las frustraciones y desarrollar habilidades para resolver los problemas que surgen en el contexto personal, familiar, social y laboral (Díaz, Muela, García \& Espinosa, 2011).

En este artículo de investigación se consideró relevante examinar desde el contexto educativo colombiano estas dos dimensiones de la inteligencia emocional. Para ello se seleccionó como grupo poblacional a adolescentes y jóvenes universitarios de Cartagena de Indias, una de las ciudades capitales más importante de Colombia en materia turística y comercial.

En este marco, Villalobos (2009) encontró una relación entre los intentos de suicidio y las dificultades que presentan los jóvenes universitarios para manejar sus emociones y enfrentar problemas derivados de situaciones como la terminación de una relación sentimental, peleas con seres queridos, vivir lejos de la familia, la pérdida del empleo y el divorcio de sus padres.

Para constatar todo lo anterior, los autores del presente trabajo se propusieron el objetivo de evaluar empíricamente si el autoconocimiento emocional es un factor asociado a la capacidad de autorregulación emocional de los adolescentes y jóvenes universitarios participantes de la muestra; y de ser así, especificar cuáles son los aspectos que guardan mayor relación. 


\subsection{Bases teóricas de la relación entre autoconocimiento emocional y autorregulación emocional}

El AE se define como la capacidad de ser conscientes de las emociones propias y a la habilidad para conocerlas y entenderlas (Bar-On, 1997; Bar-On, Brown, Kirkcaldy \& Thomé, 2000; Bernal, Esteban \& Ramírez, 2017). Según Salovey \& Mayer (1990), el AE posibilita a las personas identificar, detectar y descifrar emociones inmersas en gestos, imágenes, voces y artefactos culturales como también observar y reconocer sus propios sentimientos (Goleman, 2012). En tanto que Bisquerra \& Pérez (2007) lo conciben como la habilidad que permite a los seres humanos tomar conciencia de sus emociones, a partir de un proceso atencional que está interconectado con funciones evaluativas e interpretativas.

Este proceso abarca tanto el monitoreo y diferenciación de las emociones como aspectos actitudinales que van desde las expresiones emocionales hasta la manera en que se procesan las experiencias de este tipo (Rieffe, Oosterveld, Miers, Meerum \& Ly, 2008).

Todos los seres humanos tienen la capacidad de experimentar emociones, pero cuando hay imposibilidad o dificultad para identificarlas y describirlas, se genera la llamada Alexitimia. Este trastorno se manifiesta mediante problemas como: la dificultad para reconocer, usar y verbalizar las emociones, dificultad para localizar las sensaciones corporales y la presencia de una comunicación preverbal rígida con escasa mímica y pocos movimientos corporales (Yaben \& Montalvo, 1994).

Hay estudios que muestran una estrecha relación entre el autoconocimiento emocional y la capacidad de autorregular la conducta (Estrada, 2015; Extremera \& Fernández, 2003; Mayer \& Salovey, 1997). Incluso se sabe que los individuos con un nivel adecuado de autoconocimiento y manejo emocional, tienden a ser personas sinceras y auténticas, por cuanto son capaces de hablar abiertamente de sus emociones, de reconocer señales emocionales internas, de identificar sus sentimientos y de tener claros los valores que deben regir su conducta (Goleman, Boyatzis \& McKee, 2004).

Por su parte, Salovey \& Mayer (1990) conciben la ARE como la capacidad de mantener bajo control las emociones perturbadoras que desequilibran psicológicamente a los individuos. La regulación de los impulsos emocionales junto con la tolerancia al estrés son factores determinantes para que las personas 
aprendan a vivir calmadas, a trabajar bajo presión, a ser menos impulsivas y a responder ante eventos tensionantes sin desmoronarse (Bar-On, 1997).

De este modo, la capacidad de regular las emociones es esencial para afrontar los hechos estresantes que se presentan en la vida cotidiana de una manera proactiva y resiliente, por lo que la ausencia de esta habilidad aumenta la probabilidad de que los adolescentes y jóvenes sufran de depresión, ansiedad, problemas de conducta y bajo rendimiento académico (Rodríguez, 2017; Velasco, Manzano, Castillo \& Rovira, 2004).

En efecto, Santoya-Montes \& Garnica (2002) evaluaron la inteligencia emocional de 356 estudiantes universitarios entre los 15 y 22 años de edad, encontrando puntajes bajos en los niveles de autoconocimiento y autorregulación emocional de esta población. En cambio, los estudiantes que disfrutan de un mayor equilibrio emocional tienden a presentar mayores habilidades para identificar, comprender y regular sus emociones (Morales \& López, 2009). De esta breve revisión de antecedentes investigativos se desprende la primera hipótesis de este trabajo:

Hipótesis 1: Los niveles de autoconocimiento emocional están asociados significativamente con los niveles de autorregulación emocional de los adolescentes y jóvenes universitarios.

Por otra parte, la literatura científica sobre inteligencia emocional ha mostrado que hay diferencias en los niveles de autoconocimiento y autorregulación emocional con respecto a las características personales de los individuos. El estudio realizado por Calvo, Solórzano, Morales, Kasse, Codesal, Blanco \& Gallego (2014) con mujeres que padecen Trastornos de Conducta Alimentaria (TCA), encontró que las pacientes adultas tenían un mayor nivel de alexitimia que las adolescentes, concluyendo así que el déficit de autoconocimiento emocional se relaciona con la regulación emocional al inicio del trastorno, y que su agravamiento posterior indica una relación bidireccional entre dificultades emocionales y TCA.

Otros estudios han encontrado diferencias significativas entre los problemas de alexitimia y la edad, mostrando para el caso de los hombres un aumento significativo a partir de los 55 años en las dificultades para identificar las emociones; aunque llama la atención que los niveles de alexitimia disminuyen cuando aumenta el nivel de estudio (Garaigordobil, 2013). 
No obstante, esta tendencia parece ser circunstancial, pues en los análisis realizados a grupos de internautas no se encontraron diferencias significativas entre la edad y los niveles de autoconocimiento y autorregulación emocional (Aquino, 2003). Algo similar hallaron Ibáñez, Pérez \& Cerón (2011) al aplicar una prueba de inteligencia emocional a adolescentes escolarizados, dado que la prueba no arrojó ninguna diferencia significativa entre la edad y la inteligencia emocional.

En cuanto al género, se ha encontrado que las mujeres tienden a presentar mayores niveles de conocimiento de sus emociones que los hombres, especialmente en la forma de describir las emociones y en explicar las cosas que sienten y viven (Adams, Kuebli, Boyle \& Fivush, 1995; Feldman, Lane, Sechrest \& Schwartz, 2000). De forma similar, un estudio con 338 trabajadores del sector industrial y de servicios encontró al respecto que las mujeres presentaron puntajes superiores que los hombres en los niveles de expresividad y en la regulación de sus emociones (Gartzia, Aritzeta, Balluerka \& Barberá, 2012).

Pérez y Castejón (2005) reafirman estos hallazgos con población universitaria, al encontrar que las mujeres tuvieron mayor capacidad que los hombres para sentir y expresar sus sentimientos. En este estudio, los hombres únicamente lograron puntajes superiores que las mujeres en cuanto a la capacidad de autocomprensión de sentimientos y en la habilidad para autorregular los estados emocionales; sin embargo, el estudio realizado por Cejudo y Latorre (2015) encontró que los hombres obtuvieron mayores puntajes en las habilidades de la inteligencia emocional.

Con respecto al estrato social, hay estudios que vinculan la alexitimia con el nivel socioeconómico y cultural de las personas (Kauhanen, Kaplan, Julkunen, Wilson \& Salonen, 1993). De todas maneras, es importante mencionar casos donde no se ha encontrado ninguna influencia o asociación entre estas variables (Taylor, Ryan \& Bagby, 1985; Parker, Taylor \& Bagby, 1989).

En cuanto al tipo de institución, el estudio de Sotil, Escurra, Huerta, Rosas, Campos \& Llaños (2008) con menores de edad escolarizados, no encontró diferencias significativas entre la inteligencia emocional y el hecho de estar vinculado académicamente a una institución oficial o privada. Sin embargo, hay evidencia empírica que muestra diferencias significativas entre los niveles de inteligencia emocional de los adolescentes que estudian en 
colegios mixtos, y los que estudian en colegios de mujeres (Rosas, 2010). Estos antecedentes investigativos permitieron la formulación de la segunda hipótesis de este estudio:

Hipótesis 2: El autoconocimiento emocional y la autorregulación emocional presentan diferencias significativas asociadas con las características sociodemográficas (género, edad y tipo de universidad) de los adolescentes y jóvenes universitarios.

\section{MÉTODO}

\subsection{Diseño}

Este estudio fue de tipo cuantitativo correlacional y se basó en un diseño transversal.

\subsection{Participantes}

La población objeto de estudio la constituyen adolescentes y jóvenes universitarios de Cartagena de Indias (Colombia). La muestra fue de tipo intencional y estuvo conformada por 356 estudiantes entre los 15 y 22 años de edad que cursan carreras profesionales en tres universidades de Cartagena reconocidas oficialmente por el Ministerio de Educación Nacional.

Los rangos de edades evaluados se distribuyeron según las etapas del desarrollo propuestas por Frias, Vásquez, Del Real, Sánchez \& Servén (2012), así como por Papalia \& Wendkos (1997), quienes agrupan en la etapa de adolescencia intermedia las edades comprendidas entre los 15 y 17 años de edad, como adolescencia tardía a quienes están entre los 18 y 19 años de edad y como adultez temprana, a los que están entre los 20 y 22 años de edad. El proceso de selección de la muestra se hizo atendiendo los siguientes criterios:

Estudiantes matriculados oficialmente en tres universidades de Cartagena, de las cuales dos son de carácter privado y una pública.

Representación de estudiantes de ambos géneros (de los 356 participantes, el $48 \%$ son hombres y el $52 \%$ mujeres).

Participación de estudiantes de los distintos estratos sociales de la ciudad (el $43 \%$ de los universitarios participantes provenían de barrios de estrato bajo, el $42 \%$ de barrios de estrato medio y el $15 \%$ de barrios de estrato alto). 


\subsection{Instrumentos}

Para la recolección de la información se aplicó el cuestionario CARE, que evalúa la inteligencia emocional a partir de dos escalas o dimensiones: autoconocimiento emocional y autorregulación emocional. Este instrumento fue creado y validado por Santoya-Montes \& Garnica (2002) con población universitaria colombiana.

\subsubsection{El cuestionario CARE}

Está dividido en dos partes. La primera contiene información sociodemográfica relacionada con el género, la edad, el estrato socioeconómico y el tipo de universidad. La segunda parte del cuestionario contiene doce reactivos que miden autoconocimiento y autorregulación emocional. Los reactivos fueron medidos como variables ordinales tipo Likert que contienen opciones de respuesta que oscilan entre 1 y 4 ( 1 = muy en desacuerdo; 2 = en desacuerdo; 3 = de acuerdo; 4 = muy de acuerdo).

Los reactivos de este cuestionario están agrupados en siete factores que explicaremos a continuación. La escala de autoconocimiento emocional presenta una consistencia interna aceptable con un alfa de Cronbach de 0.72 y está compuesta por cuatro factores, a saber: percepción e identificación de sentimientos y emociones (media=2.93; desviación=0.657), capacidad para hablar abiertamente de las emociones (media=2.56; desviación=0.623), capacidad para etiquetar los sentimientos y emociones (media=3.06; desviación=0.795) y capacidad para reconocer señales emocionales internas (media=3.12; desviación=0.808).

La Escala de Autorregulación Emocional presenta también una consistencia interna aceptable con un alfa de Cronbach de 0.70 y está compuesta por tres factores que son: regulación de la impulsividad (media=3.04; desviación=0.689), estrategias de afrontamiento (media=2.78; desviación=0.724) y tolerancia a la frustración para prevenir estados emocionales negativos como ira, estrés, ansiedad y depresión (media=2.87; desviación=0.677).

Los valores de los alfas de Cronbach de ambas escalas son apropiados, teniendo en cuenta que la literatura científica sugiere aceptar los alfas mayores a 0.70 para las investigaciones aplicadas y de nivel básico (Kaplan \& Sacuzzo, 2012; Oviedo \& Campo, 2005; Yela, 1987). En la Tabla 1 se muestra la distribución de los doce reactivos que conforman los siete factores del cuestionario: 
Tabla 1

Distribución de factores y reactivos que componen el cuestionario CARE

\begin{tabular}{|c|c|c|}
\hline VARIABLES & FACTORES & REACTIVOS \\
\hline \multirow{4}{*}{$\begin{array}{l}\text { Autoconocimiento } \\
\text { emocional (AE) }\end{array}$} & $\begin{array}{l}\text { Percepción e identificación de senti- } \\
\text { mientos y emociones. }\end{array}$ & $\begin{array}{l}\text { Comprendo bien la causa de mis emociones. } \\
\text { Puedo evaluar con facilidad mis sentimientos a tal } \\
\text { punto de saber cómo me estoy sintiendo. }\end{array}$ \\
\hline & $\begin{array}{l}\text { Hablar abiertamente de las } \\
\text { emociones. }\end{array}$ & $\begin{array}{l}\text { Sé expresarles a los demás la manera como me } \\
\text { siento. } \\
\text { Los demás comprenden fácilmente lo que quiero } \\
\text { expresar. }\end{array}$ \\
\hline & $\begin{array}{l}\text { Etiquetar los sentimientos y } \\
\text { emociones. }\end{array}$ & $\begin{array}{c}\text { Cuando estoy triste o deprimido sé cuáles son las } \\
\text { razones. }\end{array}$ \\
\hline & Reconocer señale s internas. & $\begin{array}{c}\text { Reconozco la diferencia entre sentirme enojado, } \\
\text { triste o estresado. }\end{array}$ \\
\hline \multirow{4}{*}{$\begin{array}{l}\text { Autorregulación emo- } \\
\text { cional ( ARE) }\end{array}$} & $\begin{array}{l}\text { Regulación de la impulsividad (ira, } \\
\text { violencia, comportamientos de } \\
\text { riesgo). }\end{array}$ & $\begin{array}{l}\text { Con frecuencia lastimo a otros con mis actitudes o } \\
\text { mis palabras. } \\
\text { No sé escuchar a los demás, y les interrumpo } \\
\text { constantemente. }\end{array}$ \\
\hline & \multirow{2}{*}{ Estrategias de afrontamiento. } & $\begin{array}{l}\text { Cuando estoy de mal genio, no me controlo y suelo } \\
\text { ofender a otras personas. }\end{array}$ \\
\hline & & $\begin{array}{l}\text { Con frecuencia lastimo a otros con mis actitudes o } \\
\text { mis palabras. }\end{array}$ \\
\hline & $\begin{array}{l}\text { Tolerancia a la frustración para pre- } \\
\text { venir estados emocionales negativos } \\
\text { (ira, estrés, ansiedad, depresión). }\end{array}$ & $\begin{array}{l}\text { Cuando se me acaba la paciencia me vuelvo } \\
\text { ofensivo. } \\
\text { Me irrito con facilidad. }\end{array}$ \\
\hline
\end{tabular}

\subsection{Procedimiento}

Los sujetos participantes del estudio firmaron previamente un consentimiento informado. Luego diligenciaron de forma voluntaria el cuestionario CARE (instrumento de medición de autoconocimiento y autorregulación emocional), el cual fue aplicado de manera presencial al interior de las universidades de Cartagena.

El procesamiento y análisis de los datos se realizó a través del software estadístico SPSS versión 21.0. Por el tipo de variable usado en esta investigación que no tiende a presentar distribución normal, se consideró pertinente trabajar con estadística no paramétrica para contrastar las hipótesis.

Para definir los niveles de autoconocimiento emocional y autorregulación emocional, se analizaron las puntuaciones directas obtenidas por los estudiantes en cada escala. En este sentido, en el cuestionario CARE se definen como nivel bajo de autoconocimiento emocional las puntuaciones entre 6 y 10. En el nivel medio o moderado, se ubican las puntuaciones entre 11 y 15 . En el nivel medio alto están las puntuaciones entre 16 y 20 . Y en el nivel alto se sitúan las puntuaciones entre 21 y 24. 
Para comprobar la segunda hipótesis se aplicaron las pruebas de Chi-Cuadrado y Spearman y, en concreto, para establecer el grado de asociación estadística entre los niveles de autoconocimiento y autorregulación emocional y las características sociodemográficas de los participantes del estudio (género, edad, estrato, tipo de universidad donde estudian). Además, se consideró relevante evaluar las diferencias significativas entre esas características y los factores que componen las escalas de autoconocimiento y autorregulación emocional, con el fin de determinar los aspectos específicos de esos factores que presentaron un mayor peso diferencial.

Para comprobar las diferencias significativas entre la edad (adolescencia intermedia, adolescencia tardía y adultez temprana), el estrato socioeconómico (bajo, medio, alto) y los factores que componen las variables autoconocimiento emocional y autorregulación emocional, se aplicó la prueba estadística de Kruskall Wallis.

En otros casos se aplicó, por último, la prueba de Mann Whitney para comprobar si existían diferencias significativas entre los factores de estas escalas y las variables de género (hombre, mujer) y tipo de universidad (pública y privada).

\section{RESULTADOS}

\subsection{Diferencias en el autoconocimiento emocional}

Tabla 2

Nivel de autoconocimiento emocional de los participantes

\begin{tabular}{|c|c|c|c|c|c|}
\hline & & $\begin{array}{l}\text { BAJO (PUNTUA- } \\
\text { CIÓN ENTRE 6-10) }\end{array}$ & $\begin{array}{l}\text { MEDIO (PUNTUA- } \\
\text { CIÓN ENTRE 11-15) }\end{array}$ & $\begin{array}{c}\text { MEDIO ALTO (PUN- } \\
\text { TUACIÓN ENTRE } \\
16-20)\end{array}$ & $\begin{array}{l}\text { ALTO (PUNTUA- } \\
\text { CIÓN ENTRE 21-24) }\end{array}$ \\
\hline \multirow{2}{*}{$\begin{array}{l}\text { Nivel de auto- } \\
\text { conocimiento } \\
\text { emocional }\end{array}$} & Frecuencia & 9 & 84 & 215 & 48 \\
\hline & Porcentaje & 2.5 & 23.6 & 60.4 & 13.5 \\
\hline
\end{tabular}

La Tabla 2 muestra que el $73.9 \%$ de los participantes presentó puntajes de autoconocimiento emocional que oscilan entre el nivel medio alto y alto. Al aplicar las pruebas de Chi-cuadrado y Spearman, se encontró al respecto que los niveles de autoconocimiento emocional presentaron diferencias significativas asociadas con el género $(p=0.036 ; g l=3)$, los rangos de edad $(r=0.120$; $p=0.024)$ y el tipo de universidad $(p=0.019 ; g l=3)$. 
En cuanto al género, las mujeres presentaron promedios superiores en los niveles de autoconocimiento emocional (media=2.87; desviación estándar=0.72) que los hombres (media $=2.82$; desviación=0.60). Con relación a la edad, los jóvenes en el rango de adultez temprana presentaron promedios superiores en los niveles de autoconocimiento emocional (media=2.92; desviación=0.60) que quienes transitan entre la adolescencia intermedia (media=2.69; desviación=0.71) y la adolescencia tardía (media=2.86; desviación=0.68).

En cuanto al tipo de institución, los estudiantes de universidades privadas presentaron promedios superiores en los niveles de autoconocimiento emocional (media $=2.87$; desviación=0.72) en comparación con los que cursan carreras profesionales en universidades públicas (media=2.72; desviación=0.64). Los datos de la Tabla 2 revelan los factores específicos de la variable autoconocimiento emocional, en la que se concentraron las diferencias significativas.

Tabla 3

Diferencias sociodemográficas con los factores de autoconocimiento emocional

\begin{tabular}{ccccc}
\hline $\begin{array}{c}\text { CARACTERÍSTICAS } \\
\text { SOCIODEMOGRÁFICAS }\end{array}$ & $\begin{array}{c}\text { PERCEPCIÓN E IDENTIFI- } \\
\text { CACIÓN DE SENTIMIEN- } \\
\text { TOS Y EMOCIONES }\end{array}$ & $\begin{array}{c}\text { HABLAR ABIER- } \\
\text { TAMENTE DE LAS } \\
\text { EMOCIONES }\end{array}$ & $\begin{array}{c}\text { ETIQUETAR LOS } \\
\text { SENTIMIENTOSY } \\
\text { EMOCIONES }\end{array}$ & $\begin{array}{c}\text { RECONOCE SEÑA- } \\
\text { LES EMOCIONALES } \\
\text { INTERNAS }\end{array}$ \\
\hline Género & 0.247 & $0.007^{* *}$ & 0.482 & 0.985 \\
Edad & $0.039^{*}$ & 0.89 & 0.326 & 0.330 \\
Estrato & 0.125 & 0.947 & 0.905 & 0.137 \\
Tipo de universidad & $0.016^{* *}$ & & & 0.146 \\
\hline
\end{tabular}

Nota: Los datos corresponden a la significancia estadística obtenida por medio de la prueba Mann-Whitney y Kruskal-Wallis. ${ }^{*} p<0.05,{ }^{*} p<0.01$.

Según los datos de la Tabla 3, el factor "percepción e identificación de sentimientos y emociones" presentó diferencias significativas asociadas con la edad y con el tipo de universidad, mientras que el factor "hablar abiertamente de las emociones" presentó diferencias significativas con respecto al género y al tipo de universidad. 
Tabla 4

Distribución de participantes según nivel de autorregulación emocional

\begin{tabular}{|c|c|c|c|c|c|}
\hline & & $\begin{array}{c}\text { BAJO } \\
\text { (PUNTUACIÓN } \\
\text { ENTRE 6-10) }\end{array}$ & $\begin{array}{c}\text { MEDIO } \\
\text { (PUNTUACIÓN } \\
\text { ENTRE 11-15) }\end{array}$ & $\begin{array}{l}\text { MEDIO ALTO } \\
\text { (PUNTUACIÓN } \\
\text { ENTRE 16-20) }\end{array}$ & $\begin{array}{c}\text { ALTO } \\
\text { (PUNTUACIÓN } \\
\text { ENTRE 21-24) } \\
\end{array}$ \\
\hline \multirow{2}{*}{$\begin{array}{l}\text { Nivel de Autorregu- } \\
\text { lación Emocional }\end{array}$} & Frecuencia & 14 & 100 & 168 & 74 \\
\hline & Porcentaje & 3.9 & 28.1 & 47.2 & 20.8 \\
\hline
\end{tabular}

Los resultados presentados en la Tabla 4 evidencian que el $68 \%$ de los participantes presentó puntajes superiores de autorregulación, que oscilan entre el nivel medio alto y alto. Ahora bien, al aplicar las pruebas de Chi-Cuadrado y Spearman, se estableció que los niveles de autorregulación emocional presentaron diferencias significativas únicamente en relación con el tipo de universidad ( $p=0.019 ; g l=3)$ y, en cambio, no presentaron ninguna relación con el género ( $p=0.547 ; g l=3$ ) ni con los rangos de edad ( $r=0.021 ; p=0.697)$.

Tabla 5

Diferencias sociodemográficas con los factores de la autorregulación emocional

\begin{tabular}{cccc}
\hline $\begin{array}{c}\text { VARIABLES } \\
\text { SOCIODEMOGRÁFICAS }\end{array}$ & $\begin{array}{c}\text { REGULACIÓN DE LA } \\
\text { IMPULSIVIDAD }\end{array}$ & $\begin{array}{c}\text { ESTRATEGIAS DE } \\
\text { AFRONTAMIENTO }\end{array}$ & $\begin{array}{c}\text { TOLERANCIA A LA } \\
\text { FRUSTRACIÓN }\end{array}$ \\
\hline Género & 0.717 & 0.288 & 0.65 \\
Edad & 0.564 & 0.334 & 0.825 \\
Estrato & 0.549 & 0.906 & 0.306 \\
Tipo de universidad & 0.232 & $0.000 *$ & $0.019 *$ \\
\hline
\end{tabular}

Los datos corresponden a la significancia estadística obtenida por medio de la prueba Mann-Whitney y Kruskal-Wallis.

$*_{p}<0.05$.

Otro hallazgo importante consistió en que a diferencia de los que estudian en universidades privadas (media=2.75; desviación=0.80), los adolescentes y jóvenes de universidades públicas presentaron promedios superiores en los niveles de autorregulación emocional (media=3.04; desviación=0.72). La Tabla 5 también muestra que en cuanto a la autorregulación emocional, los factores "estrategias de afrontamiento" y "tolerancia a la frustración" presentan diferencias significativas con respecto al tipo de universidad donde estudian los adolescentes y jóvenes participantes de la muestra. 


\subsection{Correlación entre autoconocimiento emocional y autorregulación} emocional

Tabla 6

Correlación entre factores del autoconocimiento y la autorregulación emocional

\begin{tabular}{|c|c|c|c|c|}
\hline & & $\begin{array}{c}\text { TOLERAN- } \\
\text { CIA A LA } \\
\text { FRUSTRACIÓN }\end{array}$ & $\begin{array}{c}\text { ESTRATEGIAS } \\
\text { DE AFRONTA- } \\
\text { MIENTO }\end{array}$ & $\begin{array}{l}\text { REGULACIÓN } \\
\text { DE IMPULSOS } \\
\text { EMOCIONALES }\end{array}$ \\
\hline \multirow[t]{3}{*}{$\begin{array}{l}\text { Factores de autoconoci- } \\
\text { miento emocional }\end{array}$} & $\begin{array}{c}\text { Percepción e Identificación } \\
\text { de emociones }\end{array}$ & $0.131^{*}$ & & \\
\hline & $\begin{array}{c}\text { Hablar abiertamente de las } \\
\text { emociones }\end{array}$ & $0.154^{* *}$ & $0.140 * *$ & $0.111^{*}$ \\
\hline & $\begin{array}{l}\text { Reconocimiento de señales } \\
\text { emocionales internas }\end{array}$ & $0.136^{*}$ & $0.108^{*}$ & $0.133^{*}$ \\
\hline
\end{tabular}

Los datos corresponden a la significancia estadística obtenida por medio de la correlación de Spearman. $*_{p}<0.05, * * p<0.01$.

Al aplicar la prueba de Spearman, se encontró que los niveles de autoconocimiento emocional correlacionan significativamente y en sentido positivo con los niveles de autorregulación emocional ( $r=0.130 ; p<0.05)$. La Tabla 6 muestra, en efecto, los factores específicos de ambos niveles que están asociados significativamente.

Los datos presentados en la Tabla 6 muestran, además, que el factor "percepción e identificación de emociones" correlaciona significativamente y en sentido positivo con el factor "tolerancia a la frustración". La misma tabla registra que los factores "hablar abiertamente de las emociones" y "reconocimiento de señales emocionales internas" correlacionan significativamente y en sentido positivo con los factores "tolerancia a la frustración", "estrategias de afrontamiento" y "regulación de los impulsos emocionales".

El factor "hablar abiertamente de las emociones", que se relaciona con la dimensión comunicacional de la inteligencia emocional, mostró, finalmente, coeficientes de correlación significativos más altos que el resto de factores, aumentando así la probabilidad de que los estudiantes universitarios participantes regularan mejor sus emociones.

\section{DISCUSIÓN}

En cuanto a la primera hipótesis, se estableció que los niveles de autoconocimiento emocional de los adolescentes y jóvenes universitarios que parti- 
ciparon en este estudio están asociados significativamente con los niveles de autorregulación emocional, es decir, que a mayor autoconocimiento emocional, mayor probabilidad de autorregulación emocional. Este hallazgo es congruente con lo reportado en otras publicaciones sobre el tema (Agulló, Parra \& Bueno, 2017; Calvo et al., 2014; Estrada, 2015), que muestran que estos constructos están íntimamente relacionados, y que son aspectos determinantes en el desarrollo de la inteligencia emocional.

En el mismo orden de ideas, se encontró que, a mayor percepción e identificación de las emociones, también es mayor la probabilidad de que estos adolescentes y jóvenes desarrollen tolerancia a la frustración. De igual manera, se concluyó que la capacidad para hablar abiertamente de las emociones y de reconocer señales emocionales internas, aumenta la probabilidad de regular los impulsos emocionales, así como de generar estrategias de afrontamiento emocional y de tener mayor tolerancia a la frustración. Estos resultados reafirman desde Colombia lo encontrado por Díaz et al., (2011) y Ermer, Kahn, Salovey \& Kiehl (2012), quienes, de forma similar, afirman que las habilidades para identificar sentimientos y reconocer emociones inciden en la capacidad de desarrollar mejores niveles de autorregulación emocional.

En lo concerniente a la segunda hipótesis, se encontró que los niveles de autoconocimiento emocional presentaron diferencias significativas con respecto a la edad y el género. Estas diferencias se concentraron con mayor fuerza en la capacidad de hablar abiertamente de las emociones y de percibir e identificar los sentimientos y emociones.

Con respecto a la edad, los participantes situados en el rango de la adultez temprana presentaron mayores niveles de autoconocimiento emocional que los que se hallan en las etapas de adolescencia temprana y tardía. Dicho resultado es similar al encontrado por Martínez (2017), quien identificó diferencias estadísticamente significativas según la edad en los niveles de inteligencia emocional. De igual manera, Garaigordobil (2013) coincide en afirmar que entre más avanzamos en nuestro ciclo de desarrollo, mayor es la capacidad para evaluar las emociones, especialmente si se transita de la adolescencia a la adultez. Esto precisamente se corroboró con este estudio.

En el caso de la variable género, las mujeres presentaron mayores niveles de autoconocimiento emocional que los hombres, lo cual es congruente con los trabajos de Adams et al. (1995) y Feldman et al. (2000), quienes indican que 
las mujeres superan a los hombres en el conocimiento de sus emociones y en la capacidad de comunicarlas o hablar abiertamente de ellas.

De otro lado, no se encontraron diferencias significativas entre el estrato y los niveles de autoconocimiento y autorregulación emocional, lo cual es congruente con otras investigaciones que tampoco encontraron relación entre las características socioeconómicas y el manejo o conocimiento de las emociones (Taylor et al., 1985; Parker et al., 1989; Ibáñez et al., 2011). No obstante, conviene resaltar como nuevo hallazgo, las diferencias significativas halladas entre el tipo de institución y los niveles de autoconocimiento y autorregulación emocional.

En este sentido, es posible concluir desde la hipótesis que el AE está asociado significativamente con los niveles de ARE de los adolescentes y jóvenes universitarios, dado que los niveles de $A E$ de los sujetos participantes tienden a aumentar a medida que avanzan de la adolescencia a la adultez. Es claro, como queda dicho, que las mujeres participantes mostraron mejor capacidad comunicativa para hablar de sus emociones en comparación con los hombres, pero la autorregulación emocional no presentó ninguna relación significativa con las variables de género y edad.

Los coeficientes de correlación significativos presentados en la última tabla indican que la comunicación de las emociones son un aspecto determinante para que estos jóvenes regulen sus emociones y enfrenten mejor los problemas cotidianos. En otras palabras, los sujetos participantes que fueron capaces de hablar abiertamente de las emociones, como también de identificarlas y reconocerlas, tuvieron una mayor probabilidad de autorregular sus emociones en comparación con aquellos estudiantes que presentaron bajos puntajes en estos factores.

Los estudiantes de universidades privadas presentaron mayores niveles de autoconocimiento emocional que los de la pública, y estos a su vez superaron a los de la privada en los niveles de autorregulación emocional. Este último hallazgo amerita la generación de nuevas investigaciones que permitan explicar el por qué se dan esas diferencias; además, contradicen el estudio Sotil et al., (2008), quienes no encontraron diferencias significativas entre el manejo o desarrollo de la inteligencia emocional y el hecho de estar vinculado académicamente a una institución educativa oficial o privada.

Nota de autores:

El presente artículo es producto del trabajo de investigación titulado “EI 
autoconocimiento emocional como factor asociado a la autorregulación emocional en jóvenes universitarios en la ciudad de Cartagena".

\section{REFERENCIAS}

Adams, S., Kuebli, J., Boyle, P. \& Fivush, R. (1995). Gender differences in parent-child conversation about past emotions: a longitudinal investigation. Sex Roles, 33, 309-323. http://dx.doi.org/10.1007/BF01954572

Agulló, J. G. M., Parra-Meroño, M. C. \& Bueno, M. Á. B. (2017). Educación emocional en la universidad: propuesta de actividades para el desarrollo de habilidades sociales y personales. Vivat Academia, (139), 1-17. https://doi.org/10.15178/ va.2017.139.1-17

Aquino, A. (2003). Diferencias de género y edad en la inteligencia emocional de un grupo de Internautas. Lima: Universidad Inca Garcilaso de la Vega.

Ariza, M. H. R., Bonilla, N. M. C., León, J. C. O. \& Vaca, P. V. (2017). Convivencia escolar y cotidianidad: una mirada desde la inteligencia emocional. Revista Educación y Desarrollo Social, 11(1). http://dx.doi.org/10.18359/reds.2649

Bar-On, R. (1997). The Emotional quotient inventory: a test of emotional intelligence. Toronto: Multi-Health Systems.

Bar-On, R., Brown, J., Kirkcaldy, B. \& Thomé, E. (2000). Emotional expression and implications for occupational stress: an application of the Emotional Quotient Inventory (EQ-i). Personality and Individual Differences, 28, 1107-1118. http:// dx.doi.org/ 10.1016/S0191-8869(99)00160-9

Bernal, N. C., Esteban, S. S. \& Ramírez, F. C. (2014). Estimulación de la inteligencia emocional como prevención del fenómeno "bullying" en alumnos víctimas. Revista INFAD de Psicología, 6(1), 427-430. http://dx.doi.org/ 10.17060/ ijodaep.2014.n1.v6.762

Bisquerra, R. \& Pérez, N. (2007). Las competencias emocionales. Educación XXI(10), 61-82. http://dx.doi.org/ 10.5944/educxx1.1.10.297

Calvo, R., Solórzano, G., Morales, C., Kasse, M., Codesal, R., Blanco, A. \& Gallego Morales, L. (2014). Procesamiento emocional en pacientes TCA adultas vs. Adolescentes: reconocimiento y regulación emocional. Clínica y Salud, 25, 19-37.

Cejudo, J. \& Latorre, S. (2015). Effects of the Spock videogame on improving emotional intelligence in adolescents. Electronic Journal of Research in Educational Psychology, 13, 319-342. http://doi.org/10.14204/ejrep.36.15060

Díaz, M., Muela, J., García, L. \& Espinosa, L. (2011). La inteligencia emocional en adolescentes diagnosticados con ansiedad social. En P. Fernández (Ed.), Inteligencia emocional: 20 años de investigación y desarrollo (pp. 109-112). Pedrueca: Fundación Marcelino Botín.

Enríquez, M., Fajardo, M., Garzón, F. (2015). Una revisión general a los hábitos y técnicas de estudio en el ámbito universitario. Psicogente, 18(33), 166-187. http://doi.org/10.17081/psico.18.33.64

Ermer, E., Kahn, R., Salovey, P. \& Kiehl, K. (2012). Emotional intelligence in incarcerated men with psychopathic traits. Journal of Personality and Social Psychology, 103, 194-204. http://doi.org/ 10.1037/a0027328 
Estrada, N. G. G. (2015). Desarrollo de competencias emocionales en jóvenes bachilleres bajo un enfoque psicoeducativo. Caleidoscopio, 18(33), 157-180. Disponible en http://revistas.psico-ags.net/index.php/caleidoscopio/article/view/185/107

Extremera, N. \& Fernández, P. (2003). La inteligencia emocional en el contexto educativo: hallazgos científicos de sus efectos en el aula. Revista de Educación, 332, 97-116. Disponible en https://www.mecd.gob.es/revista-de-educacion/ numeros-revista-educacion/numeros-anteriores/2003/re332/re332_06.html

Extremera, N., Férnandez, P. \& Salovey, P. (2006). Spanish version of the Mayer-Salovey-Caruso Emotional Intelligence Test (MSCEIT). Version 2.0: reliabilities, age and gender differences. Psicothema, 18(1), 42-48. http://www.psicothema.com/ psicothema.asp?ID $=3274$

Feldman, B., Lane, R., Sechrest, L. \& Schwartz, G. (2000). Sex differences in emocional awareness. Personality and Social Psychology Bulletin, 26, 1027-1035. http://doi. org/10.1177/01461672002611001

Frias, A., Vásquez, M., Del Real, Á., Sánchez, C. \& Servén, E. (2012). Conducta autolesiva en adolescentes: prevalencia, factores de riesgo y tratamiento. Cuadernos de Medicina Psicosomática y Psiquiatría de Enlace, (103), 33-48 Disponible en http://www.editorialmedica.com/download.php?idart=468

Garaigordobil, M. (2013). Sexism and alexithymia: correlations and differences as a function of gender, age, and educational level. Anales de Psicología, 29, 268-377. http://dx.doi.org/10.6018/analesps.29.2.132261

Gartzia, L., Aritzeta, A., Balluerka, N. \& Barberá, E. (2012). Inteligencia emocional y género: más allá de las diferencias sexuales. Anales de Psicología, 28, 567-575. http://doi.org/10.6018/analesps.28.2.124111.

Goleman, D. (2012). Inteligencia Emocional (22 ed.). Barcelona: Bolsillo Zeta.

Goleman, D., Boyatzis, R. \& McKee, A. (2004). El líder resonante crea más. Barcelona: Plaza-Janes editores.

Ibáñez, M., Pérez, I. \& Cerón, D. (2011). Inteligencia emocional en adolescentes de dos colegios de Bogotá. Revista Colombiana de Psiquiatría, 40, 49-64. http://doi. org/10.1016/S0034-7450(14)60104-9

Kaplan, R. \& Saccuzzo, D. (2012). Psichological testing: principles, applications, and issues. Wadsworth: Cengage Learning.

Kauhanen, J., Kaplan, G., Julkunen, J., Wilson, T. \& Salonen, J. (1993). Social factors in alexithymia. Comprehensive Psychiatry 34, 330-335. http://doi. org/10.1016/0010-440X(93)90019-Z.

Lagardera, F. \& Masciano, A. (2011). Aprendiendo a desanudarse: una práctica psicomotriz introyectiva de autoconocimiento y autorregulación emocional. En memorias del XIV Seminario Internacional y II Latinoamericano de Praxiología Motríz, Educación física y contextos críticos (pp. 1-22). La Plata: Agencia Nacional de Promoción Científica y Tecnológica.

Martínez, R. S. (2017). Relación entre perfiles de inteligencia emocional y bienestar psicológico en personas con lesión medular. Anuario de Psicología, 47(1), 9-17. http://doi.org/10.1016/j.anpsic.2017.05.001

Mayer, J. \& Salovey, P. (1997). What is emotional intelligence? En P. Salovey y D. Sluyter (eds.), Emotional development and emotional intelligence: implications for educators (pp. 3-31). New York: Basic Books. 
Morales, M. \& López, E. (2009). Inteligencia emocional y rendimiento escolar: estado actual de la cuestión. Revista Latinoamericana de Psicología, 41, 69-79. http:// doi.org/10.14349/rlp.v41i1.556

Oviedo, H. \& Campo, A. (2005). Aproximación al uso del coeficiente alfa de Cronbach. Revista Colombiana de Psiquiatria, 36, 572-580.

Papalia, D. \& Wendkos, S. (1997). Desarrollo humano: con aportaciones para Iberoamérica.Bogotá: McGraw-Hill.

Parker, J., Taylor, G. \& Bagby, R. (1989). The alexithymia construct: relationship with sociodemographic variables and intelligence. Comprehensive Psychiatry 30, 434-441. http://doi.org/10.1016/0010-440X(89)90009-6

Pérez, N. \& Castejón, J. (2005). Diferencias en el perfil de Inteligencia Emocional en estudiantes universitarios de distintas titulación. Psicología Social y Problemas Sociales (1), 197-204. Disponible en http://www.educacion.udc.es/grupos/ gipdae/documentos/congreso/VIIIcongreso/pdfs/165.pdf

Rieffe, C., Broekhof, E., Kouwenberg, M., Faber, J., Tsutsui, M. \& Güroğlu, B. (2016). Disentangling proactive and reactive aggression in children using self-report. European Journal of Developmental Psychology, 13, 439-45. http://doi.org/10.10 80/17405629.2015.1109506

Rieffe, C., Oosterveld, P., Miers, A., Meerum, M. \& Ly, V. (2008). Emotion awareness and internalising symptoms in children and adolescents: the emotion awareness questionaire revised. Personality and Individual Differences, 45, 756-761. http:// doi.org/10.1016/j.paid.2008.08.001

Rodríguez, F. M. M. (2017). Relaciones entre afrontamiento del estrés cotidiano, autoconcepto, habilidades sociales e inteligencia emocional. European Journal of Education and Psychology. https://doi.org/10.1016/j.ejeps.2017.04.001

Rosas, N. (2010). Niveles de inteligencia emocional en estudiantes de quinto año de secundaria de colegios mixtos y diferenciados del callao. Lima: Universidad San Ignacio.

Salovey, P. \& Mayer, J. (1990). Emotional intelligence. Imagination, Cognition and Personality, 9(3), 185-211. http://doi.org/10.2190/DUGG-P24E-52WK-6CDG.

Santoya-Montes, Y. \& Garnica, C. (2002). Relación entre rendimiento académico e inteligencia emocional de los estudiantes de pregrado de la Universidad Tecnológica de Bolívar. Cartagena de Indias: Universidad Tecnológica de Bolívar.

Sotil, A., Escurra, L., Huerta, R., Rosas, M., Campos, E. \& Llaños, A. (2008). Efectos de un programa para desarrollar la inteligencia emocional en alumnos del sexto grado de educación primaria. Revista de Investigación en Psicología, 11, 55-65. http://dx.doi.org/10.15381/rinvp.v11i2.3819

Szczygiet, D. \& Mikolajczak, M. (2017). Why are people high in emotional intelligence happier? They make the most of their positive emotions. Personality and Individual Differences, 117, 177-181. http://doi.org/10.1016/j.paid.2017.05.051

Taylor, G., Ryan, D. \& Bagby, R. (1985). Toward the development of a new self-report alexithymia scale. Psychotherapy Psychosomatic, 44, 191-199. http://doi. org/10.1159/000287912

Valles, A. \& Valles, C. (2003). Programa para el desarrollo de la inteligencia emocional. España: El gabinete de orientación psicológica. 
Velasco, C., Manzano, M. C., Castillo, I. \& Rovira, D. (2004). Afrontamiento y regulación emocional de hechos estresantes: un meta-análisis de 13 estudios. Boletín de Psicología, 82, 25-44.

Villalobos, F. (2009). Situación de la conducta suicida en estudiantes de colegios y universidades de San Juan de Pasto, Colombia. Salud Mental, 32, 165-171.

Yaben, S. \& Montalvo, J. (1994). Alexitimia: concepto, evaluación y tratamiento. Psicothema, 6, 357-366.

Yela, M. (1987). Toward a unified psychological science: The meaning of behavior. In A. W. Staats \& L. P. Mos (Eds.), Annals of theoretical psychology, 5, 241-274. New York, NY, US: Plenum Press. autor o el licenciante.

\section{(cc) BY}

